University of Nebraska - Lincoln

DigitalCommons@University of Nebraska - Lincoln

Theses, Dissertations, and Student Research:

Department of Physics and Astronomy

Physics and Astronomy, Department of

Spring 1-16-2008

\title{
In Situ Measurement of Three-Dimensional Ion Densities in Focused Femtosecond Pulses
}

James Strohaber

Texas A \& M University - College Station, jstroha1@gmail.com

Cornelis J. Uiterwaal

University of Nebraska-Lincoln, cuiterwaal2@unl.edu

Follow this and additional works at: https://digitalcommons.unl.edu/physicsdiss

Part of the Engineering Commons, Life Sciences Commons, Medicine and Health Sciences Commons, and the Physics Commons

Strohaber, James and Uiterwaal, Cornelis J., "In Situ Measurement of Three-Dimensional Ion Densities in Focused Femtosecond Pulses" (2008). Theses, Dissertations, and Student Research: Department of Physics and Astronomy. 14.

https://digitalcommons.unl.edu/physicsdiss/14

This Article is brought to you for free and open access by the Physics and Astronomy, Department of at DigitalCommons@University of Nebraska - Lincoln. It has been accepted for inclusion in Theses, Dissertations, and Student Research: Department of Physics and Astronomy by an authorized administrator of DigitalCommons@University of Nebraska - Lincoln. 


\title{
In Situ Measurement of Three-Dimensional Ion Densities in Focused Femtosecond Pulses
}

\author{
J. Strohaber* and C. J. G. J. Uiterwaal ${ }^{\dagger}$ \\ Behlen Laboratory, Department of Physics and Astronomy, The University of Nebraska-Lincoln, \\ City Campus, Lincoln, Nebraska 68588-0111, USA \\ (Received 10 August 2007; published 16 January 2008)
}

\begin{abstract}
We image spatial distributions of $\mathrm{Xe}^{q+}$ ions in the focus of a laser beam of ultrashort, intense pulses in all three dimensions, with a resolution of $\sim 3 \mu \mathrm{m}$ and $\sim 12 \mu \mathrm{m}$ in the two transverse directions. This allows for studying ionization processes without spatially averaging ion yields. Our in situ ion imaging is also useful to analyze focal intensity profiles and to investigate the transverse modal purity of tightly focused beams of complex light. As an example, the intensity profile of a Hermite-Gaussian beam mode $\mathrm{HG}_{1,0}$ recorded with ions is found to be in good agreement with optical images.
\end{abstract}

DOI: 10.1103/PhysRevLett.100.023002

After the development of chirped pulse amplification [1] in the late 1980s and the discovery of self-mode-locking [2] in 1991, laser sources emitting femtosecond-duration pulses have become routinely available. Laboratories around the world have used such sources for extensive studies of intense-field processes such as multiphoton ionization, tunneling ionization, and above-threshold ionization of atoms and of molecules [3]. The required atomic electric field of about $10^{9} \mathrm{~V} / \mathrm{cm}$ or more is created by focusing the ultrashort pulses in a target gas.

A direct consequence of carrying out intense-field experiments with focused laser light is the presence of a broad range of peak intensities across the focal region. To understand some of the experimental consequences of this let us consider a process for which the probability to produce a certain product particle is $P(I)$ when the pulse has peak intensity $I$. Following common practice, we might study the process by adjusting the laser pulse energy such that the highest peak intensity found in the focus equals $I_{0}$ and then collecting product particles from all over the focal region. Doing so, we obtain a volumeintegrated product yield $Y\left(I_{0}\right)$ given by

$$
Y\left(I_{0}\right) \propto \int_{0}^{I_{0}} P(I)\left|\frac{d V\left(I, I_{0}\right)}{d I}\right| d I,
$$

in which $V\left(I, I_{0}\right)$ is the volume in which the local peak intensity exceeds some value $I$ [4]. Because of the volumetric weighting in Eq. (1), the measured yield $Y\left(I_{0}\right)$ differs from the probability $P(I)$ under investigation. For instance, when $I_{0}$ is increased, $Y\left(I_{0}\right)$ ultimately becomes proportional to $I_{0}^{3 / 2}$ once the saturation intensity [for which $P\left(I_{0}\right) \rightarrow 1$ ] is exceeded [5]. This masks the decrease of $P(I)$ that is often expected for intensities greater than the saturation intensity due to competing higher-order processes. Also, subtle features in $P(I)[6,7]$ tend to be washed out by volume integration.

In this Letter, we present spatially resolved images of 3D densities of ion charge states as they are created in focused ultrashort pulses. Thus, what we have realized for use in
PACS numbers: $32.80 . \mathrm{Rm}, 32.80 . \mathrm{Wr}, 41.85 . \mathrm{Ew}, 42.30 . \mathrm{Wb}$

intense-field ionization research is a photodynamical test tube, i.e., a volume of known, $\mu \mathrm{m}$-sized dimensions exposed to peak intensities that are essentially constant across the volume. This allows measuring $P(I)$ without spatial integration: one simply defines a sufficiently limited spatial region of interest in the ion image and determines the yields originating from this region.

Another goal of our investigations is to analyze focal intensity distributions. These can be inferred because volume elements having the same peak intensity contain identical ion charge state distributions. The target gas then acts as an in situ sensor of local intensity. In this Letter we analyze images, made with $\mathrm{Xe}$ ions, of the transverse profile of an intense, ultrashort focused Hermite-Gaussian $\mathrm{HG}_{0,1}$ transverse beam mode. The $\mathrm{HG}$ modes are related to the Laguerre-Gaussian modes $L G_{p, \ell}$ [8]. We are particularly interested in LG modes because they carry a sharply defined amount of optical orbital angular momentum (OAM) [9], and we wish to study the effect of this quantity on intense-field processes. For this, we need modally pure LG modes. In situ ion imaging will be helpful to assess the degree of modal purity: modal interferences due to contaminating mode components (which have different Gouy phases [8]) should show up in the ion image.

Other techniques have been reported to image foci and/ or circumvent volumetric weighting. First, the intensityselective scanning (ISS) technique [4,10-13] utilizes a narrow slit perpendicular to the laser propagation direction, so ion yields are still integrated over two dimensions. To obtain yields free of volume integration raw ISS data must be deconvolved, which adds noise and only works for Gaussian intensity distributions. Second, the intensitydifference spectrum (IDS) technique [14,15] has been shown to work only for a two-dimensional Gaussian profile, and is inherently prone to statistical noise. Last, a number of time-of-flight techniques allow ions created at different locations to arrive at different times [6,16-18]. The technique of [16] was demonstrated to work well for 
$\sim 200 \mu \mathrm{m}$ resolution [17], but more systematic investigations are required [16] to explore its applicability for resolutions of a few micrometer. The setup of [18] only achieved a spatial resolution of $0.68 \mathrm{~mm}$, and requires cold targets. A focus with a waist of a few $\mathrm{mm}$ was dissected into 12 adjacent detection volumes of a few $100 \mu \mathrm{m}$ [6].

Compared to the previous methods, the present method achieves a better spatial resolution $(\sim 3 \mu \mathrm{m}$ and $\sim 12 \mu \mathrm{m}$ in the two transverse directions of the beam). It does not require $a$ priori knowledge about the intensity distribution, and is not limited to Gaussian profiles. We show the first spatially resolved ion distributions in three dimensions with resolution of a few micrometer.

A schematic of our setup is shown in Fig. 1(a). We used an 800-nm Ti:sapphire laser system (Spectra Physics Tsunami/Spitfire) delivering $\sim 50-\mathrm{fs}$ pulses at a $1-\mathrm{kHz}$ repetition rate with an average output power of about $1.2 \mathrm{~W}$. We focus the radiation into an evacuated ionization chamber using an antireflection-coated uv-grade fused silica plano-convex lens with a nominal focal length of $22.7 \mathrm{~cm}$ for $800 \mathrm{~nm}$. We lowered the background pressure in the ionization chamber to below $10^{-9}$ mbar using a liquid- $\mathrm{N}_{2}$ trap. We then admitted $\mathrm{Xe}$ gas (purity $99.999 \%$ ) to a total pressure of $\sim 5.0 \times 10^{-7}$ mbar. To detect the $\mathrm{Xe}$ ions created in the focus, we use a reflectron-type time-of-flight (TOF) ion mass spectrometer. The focus is positioned between two parallel plates. One of these is at ground potential and contains the spectrometer entrance slit; the other plate ("repeller") carries a positive voltage $V_{R}$ of about $+1.5 \mathrm{kV}$ and is at a distance $d$

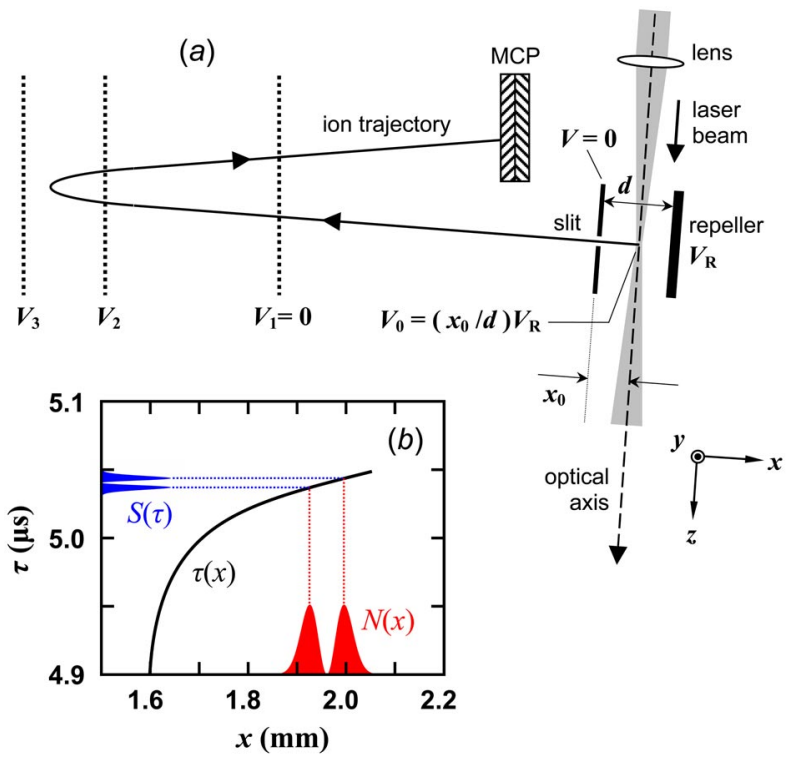

FIG. 1 (color online). (a) Schematic of our setup (not to scale). The dotted lines represent meshes held at fixed electrostatic potentials. (b) Graph of the monotonic function $\tau(x)$ as typically used in our experiments. A recorded time-of-flight spectrum $S(\tau)$ may be thought of as the "image in time" of the density $N(x)$ of ions in space. (See text for further details.) of about $3 \mathrm{~mm}$ away. The uniform electrostatic field between the plates causes target gas cations created in the focus to be accelerated toward and pass through the slit. The slit is rectangular, measuring [see coordinate system in Fig. 1(a)] $\Delta z \sim 400 \mu \mathrm{m}$ by $\Delta y \sim 12 \mu \mathrm{m}$. Assuming their initial kinetic energy is negligible, ions created at a distance $x$ away from the slit enter the TOF tube with kinetic energy proportional to $(x / d) V_{R}$. The total TOF of an ion with mass $m=M u\left(u\right.$ is 1 a.m.u. $\left.\approx 1.67 \times 10^{-27} \mathrm{~kg}\right)$ and charge $q=Q e(e$ is the elementary charge $\approx 1.60 \times$ $10^{-19} \mathrm{C}$ ) equals

$$
t=\sqrt{\frac{M}{Q}} \tau(x)
$$

In this equation, $\tau(x)$ is a reference TOF of an ion with $M=Q$ (e.g., $\mathrm{H}^{+}$) that was created at position $x$; this function depends on the electrostatic potentials $V_{2}$ and $V_{3}$ we apply to the meshes [see Fig. 1(a); note that $V_{1}=$ $0 \mathrm{~V}]$. The ion trajectories inside the reflectron have a turning point, and the ions are detected on a multi-channelplate ion detector [multichannel plate (MCP) in Fig. 1(a)], a Galileo chevron type with a time resolution of $\Delta t \approx$ $1.5 \mathrm{~ns}$. To record the ion signal from the MCP we used a FAST ComTec model P7886 2-GHz counting card with time bins of 500 ps.

To reconstruct three-dimensional ion distributions, we note that the entrance slit already clips the acceptance volume in the two dimensions $y$ and $z$ to the sizes $\Delta y$ and $\Delta z$ of the slit. We reconstruct the initial positions of ions in the third dimension $(x)$ from their TOF spectrum [Eq. (2)]. To obtain a one-to-one correspondence between initial location $x$ of an ion and its TOF for each $M / Q$ ratio, we adjust the mesh potentials $V_{2}$ and $V_{3}$ so that a monotonically increasing $\tau(x)$ results. Typical values are $V_{2}=$ $815 \mathrm{~V}$ and $V_{3}=1048 \mathrm{~V}$; a graph of $\tau(x)$ for these values is shown Fig. 1(b). [When $V_{2}$ is lowered to $\sim 800 \mathrm{~V}, \tau(x)$ becomes flat, i.e., independent of $x$ over a wide range. For such a setting there is no longer a one-to-one correspondence between $x$ and TOF, so spatial information would be lost.] For a fixed accumulation time, the signal $S_{j}$ recorded in time bin $j$ is proportional to the number of ions with TOF between $t_{j}=j \Delta t$ and $t_{j+1}=(j+1) \Delta t$ ( $\Delta t$ is time bin width). These ions must all originate from a small spatial interval $\Delta x_{j}=x_{j+1}-x_{j}$, with $t_{j}=\sqrt{M / Q} \tau\left(x_{j}\right)$ and $t_{j+1}=\sqrt{M / Q} \tau\left(x_{j+1}\right)$. The density of ions $N_{j}$ created in the spatial interval $\Delta x_{j}$ is thus

$$
N_{j} \propto \frac{\Delta t_{j}}{\Delta x_{j}} S_{j} \propto \frac{S_{j}}{\Delta x_{j}} .
$$

The second proportionality follows because all $\Delta t_{j}$ are equal. We may think of the recorded TOF spectrum $S_{j}$ as the image in time of the density $N_{j}$ of ions in space. The spatial resolution (in the $x$ direction) is 


$$
\Delta x_{j} \approx \sqrt{\frac{Q}{M}} \frac{\Delta t}{(\partial \tau / \partial x)_{x=x_{j}}} .
$$

For the typical settings we used, the derivative $\partial \tau / \partial x$ smoothly drops from about $0.250 \mathrm{~ns} / \mu \mathrm{m}$ (at $x=$ $1.9 \mathrm{~mm}$ ) to about $0.200 \mathrm{~ns} / \mu \mathrm{m}$ (at $x=2.0 \mathrm{~mm}$ ). To measure $\tau(x)$ we used a focused attenuated beam, which generates only $\mathrm{Xe}^{+}$. We positioned this focus at various $x$ positions using a translation stage with $\mu \mathrm{m}$ precision, and recorded the TOF of the $\mathrm{Xe}^{+}$. The obtained experimental $\tau(x)$ matched the theoretically predicted curve. Using Eq. (4) with a time resolution $\Delta t=1.5 \mathrm{~ns}$ as set by our MCP, we find our theoretical spatial resolution for ${ }^{132} \mathrm{Xe}^{+}$ranges between $0.52 \mu \mathrm{m}$ (at $1.9 \mathrm{~mm}$ ) and $0.66 \mu \mathrm{m}$ (at $2.0 \mathrm{~mm}$ ). For ${ }^{132} \mathrm{Xe}^{7+}$, these values would be 1.4 and $1.7 \mu \mathrm{m}$, respectively. A more realistic estimation of the spatial resolution accounts for the thermal motion of the Xe gas, the dimensions of the spectrometer entrance slit, mechanical vibrations, and the frequency bandwidth of our detection electronics. A first analysis of these factors indicates the spatial resolution in the $x$ direction is about $3 \mu \mathrm{m}$. In the $y$ and $z$ directions the resolutions are mainly determined by the slit dimensions, so 12 and $400 \mu \mathrm{m}$, respectively. Slits narrower than $12 \mu \mathrm{m}$ could be used, but the resolution in the $y$ dimension is ultimately limited by thermal effects, in particular for the lower charge states; this limits our technique to laser foci not smaller than about $10 \mu \mathrm{m}$. We chose the $400-\mu \mathrm{m}$ slit length as a compromise between count rate and the need to keep the slit shorter than the Rayleigh range of our focused beam, which is a few millimeters. Pressure-dependent measurements indicated that space charge effects are absent for the pressure we used $\left(\sim 5.0 \times 10^{-7}\right.$ mbar $)$.

Figures 2 and 3 show ${ }^{132} \mathrm{Xe}$ ion distributions in the focus reconstructed using Eqs. (3) and (4). The different charge states are color coded: $\mathrm{Xe}^{+}$in red (gray in print), $\mathrm{Xe}^{2+}$ in green (light gray), $\mathrm{Xe}^{3+}$ in blue (dark gray), and $\mathrm{Xe}^{4+}$ in red (gray). The image in Fig. 2 is a cross section in the $x y$ plane through the focal region, obtained by moving the focus up ( $y$ direction) in steps of $10 \mu \mathrm{m}$ using a stepper motor, and recording a TOF spectrum for each height. The image in Fig. 3 is a cross section in the $x z$ plane, obtained by moving the focus along the $z$ direction in steps of $200 \mu \mathrm{m}$. Setting up the experiment, we noticed that visible imperfections in the unfocused beam profile go hand in hand with irregular features in the ion charge state distributions. Using this as a guideline, we improved the mode quality of the laser by reducing the aperture of an iris in the regenerative cavity of the amplifier, at the expense of $\sim 0.1 \mathrm{~W}$. The nesting of the various charge states in Figs. 2 and 3 indicates the competition between the processes that create them. The four line graphs in Fig. 2 show the ion yields measured along the dashed line in Fig. 2 (transverse to the laser propagation, same scale). If one moves toward the center of the focus, the yield of each

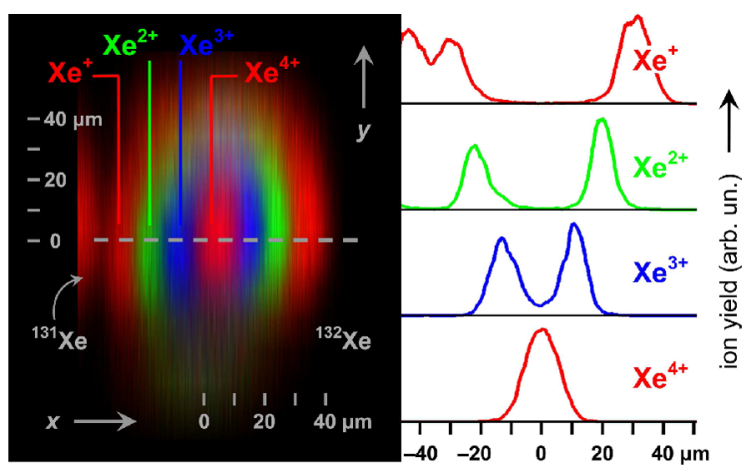

FIG. 2 (color online). Measured distribution of ${ }^{132} \mathrm{Xe}$ ions in an $x y$ plane through the focus, with $\mathrm{Xe}^{+}$in red (gray in print), $\mathrm{Xe}^{2+}$ in green (light gray), $\mathrm{Xe}^{3+}$ in blue (dark gray), and $\mathrm{Xe}^{4+}$ in red (gray), as indicated. The isotope 132 was used because it is the most abundant. The signal on the left is due to ${ }^{131} \mathrm{Xe}^{+}$. Cross sections along the dashed line are shown on the right. The observed nesting of the charge states occurs because the peak intensity is higher at locations closer to the center of the focus. A charge state that dominates at lower-intensity locations is depleted in favor of the next-higher charge state at higher-intensity locations.

charge state $\mathrm{Xe}^{q+}$ rises, saturates, and is then depleted in favor of the next charge state $\mathrm{Xe}^{(q+1)+}$ in a sequential way, so that a nested pattern of charge states results. The depletion shown in Fig. 2 is in sharp contrast with the 3/2-power rise observed in traditional methods. From the data shown

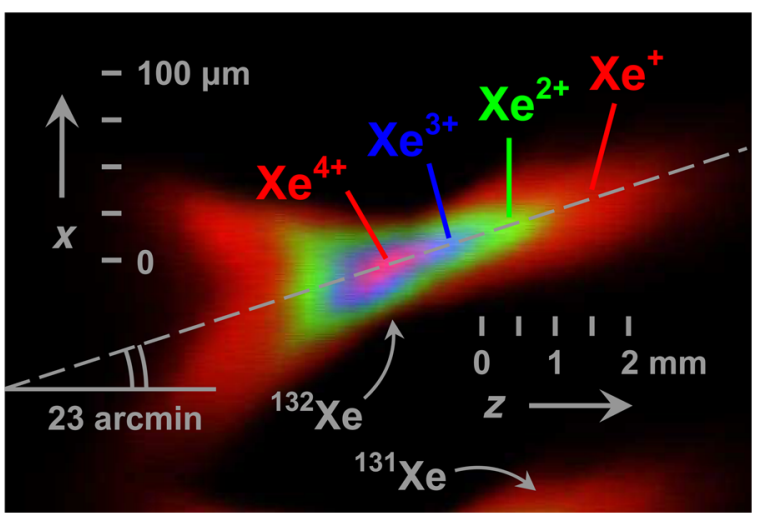

FIG. 3 (color online). Measured distribution of ${ }^{132} \mathrm{Xe}$ ions in the $x z$ plane, with $\mathrm{Xe}^{+}$in red (gray in print), $\mathrm{Xe}^{2+}$ in green (light gray), $\mathrm{Xe}^{3+}$ in blue (dark gray), and $\mathrm{Xe}^{4+}$ in red (gray), as indicated. The dashed line is the optical axis (beam propagates to the right). Note the difference in scale between the $x$ and $z$ axes. The small angle of 23 arcmin between the optical axis and the $z$ axis is due to a slight misalignment of our setup. The signal at the bottom of the image is due to ${ }^{131} \mathrm{Xe}$ ions. The observed nesting of the charge states occurs because the peak intensity is higher at locations closer to the center of the focus. A charge state that dominates at lower-intensity locations is depleted in favor of the next-higher charge state at higher-intensity locations. To avoid overlap between adjacent isotopes we used slightly different voltages than those used to obtain Fig. 2. 


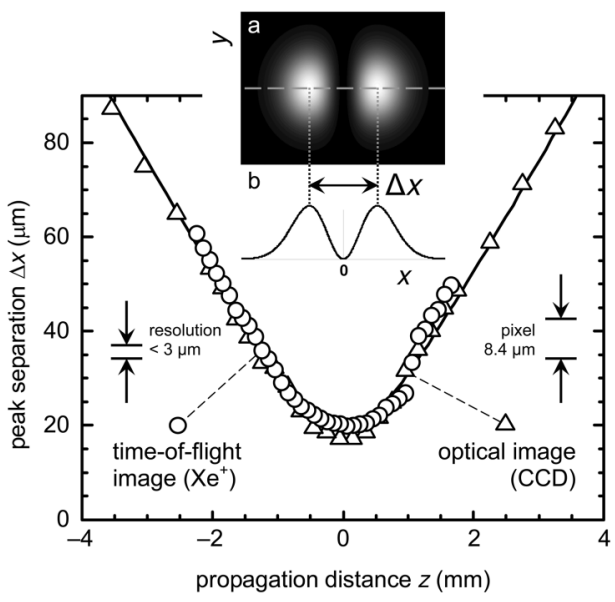

FIG. 4. Focused $\mathrm{HG}_{1,0}$ beam: distance $\Delta x$ between the two intensity lobes (see insets) as a function of the propagation distance $z$. Circles: from $\mathrm{Xe}^{+}$image (resolution $<3 \mu \mathrm{m}$ ); triangles: from CCD image (pixel size $8.4 \mu \mathrm{m}$ ). Solid curve: fit to Eq. (6).

in the line graphs, we measured that the count rate of $\mathrm{Xe}^{+}$ and $\mathrm{Xe}^{2+}$ in the center of the focus is only a few percent of that of the peaks. Such a degree of observed depletion has no precedence in literature, and underlines our ability to record $P(I)$ free of volumetric weighting.

About $5 \times 10^{6}$ laser shots (or a runtime of about $2 \mathrm{~h}$ including overhead activities) were required to produce each of Figs. 2 and 3. Ion count rates for the line graphs were a few hundred per minute per time channel.

To demonstrate that our ion imaging technique is not limited to only Gaussian intensity profiles, we also imaged a focused beam of ultrashort pulses in the HermiteGaussian $\mathrm{HG}_{1,0}$ transverse mode [8] (Fig. 4). We created this mode using holographic techniques $[19,20]$. The intensity of the $\mathrm{HG}_{1,0}$ mode is

$$
I(x, y, z) \propto \frac{x^{2}}{w(z)^{2}} \exp \left(-\frac{x^{2}+y^{2}}{w(z)^{2}}\right),
$$

in which $w(z)^{2}=w_{0}^{2}\left(1+z^{2} / z_{0}^{2}\right)$ describes the self-similar transverse scaling of the intensity profile ( $w_{0}$ is the beam waist and $z_{0}$ is the Rayleigh range) [8]. The profile consists of two lobes (see inset $a$ in Fig. 4). For $y=0$ (dashed line in inset) the maxima of these lobes are separated in the $x$ direction by a distance

$$
\Delta x(z)=\Delta x_{0} \sqrt{1+z^{2} / z_{0}^{2}}
$$

(see inset $b$ ), with $\Delta x_{0}$ the separation at the waist $(z=0)$. Again using Eqs. (3) and (4), we reconstructed $\Delta x(z)$ (circles in Fig. 4) from TOF spectra.

To verify that this reconstruction is correct we also focused the beam in air using the same lens as in the ionization experiment, and recorded the beam profile as a function of $z$ optically (triangles in Fig. 4) using a CCD camera. In contrast to the ion imaging experiments - taken at full power - this optical experiment required substantial attenuation, to protect the camera from damage and to avoid nonlinear optical effects in air. The ion image and optical image agree within the error limits, which we estimate to be $\sim 8.4 \mu \mathrm{m}$ (pixel size) for the CCD data and $\sim 3 \mu \mathrm{m}$ for the ion data. This agreement allows a fit to Eq. (6) (solid curve in Fig. 4).

In summary, we used a time-of-flight technique to image ion distributions in the focus of a laser beam of ultrafast pulses. We showed that this (a) allows measurements of ion yields without volumetric weighting, and (b) facilitates investigations of focal intensity distributions, with the target atoms acting as in situ intensity sensors.

This material is based upon work supported by the National Science Foundation under Grant No. PHY0355235. The authors gratefully acknowledge the MaxPlanck-Institute of Quantum Optics in Germany, in particular Dr. Hartmut Schröder, for generously lending us the reflectron used in this work.

*jstroha1@ @igred.unl.edu †cuiterwaal2@unl.edu

[1] M. Pessot, P. Maine, and G. Mourou, Opt. Commun. 62, 419 (1987).

[2] D. E. Spence, P. N. Kean, and W. Sibbett, Opt. Lett. 16, 42 (1991).

[3] J. H. Posthumus, Rep. Prog. Phys. 67, 623 (2004).

[4] M. A. Walker, P. Hansch, and L. D. Van Woerkom, Phys. Rev. A 57, R701 (1998).

[5] S. Speiser and J. Jortner, Chem. Phys. Lett. 44, 399 (1976).

[6] R. R. Jones, Phys. Rev. Lett. 74, 1091 (1995).

[7] A. Talebpour, C.-Y. Chien, and S. L. Chin, J. Phys. B 29, 5725 (1996).

[8] A.E. Siegman, Lasers (University Science Books, Sausalito, CA, 1986).

[9] Optical Angular Momentum, edited by L. Allen, S. M. Barnett, and M.J. Padgett (Institute of Physics Publishing, Bristol, U.K., 2003).

[10] P. Hansch, M. A. Walker, and L. D. Van Woerkom, Phys. Rev. A 54, R2559 (1996).

[11] S. Banerjee, G. Ravindra Kumar, and D. Mathur, J. Phys. B 32, L305 (1999).

[12] L. Robson et al., J. Am. Soc. Mass Spectrom. 16, 82 (2005).

[13] W. A. Bryan et al., Phys. Rev. A 73, 013407 (2006).

[14] P. Q. Wang et al., Opt. Lett. 30, 664 (2005).

[15] E. P. Benis et al., Phys. Rev. A 70, 025401 (2004).

[16] B. Witzel et al., Int. J. Mass Spectrom. Ion Processes 172, 229 (1998).

[17] B. Witzel et al., Phys. Rev. A 58, 3836 (1998).

[18] R. Bredy et al., J. Opt. Soc. Am. B 21, 2221 (2004).

[19] I. G. Mariyenko, J. Strohaber, and C. J. G. J. Uiterwaal, Opt. Express 13, 7599 (2005).

[20] J. Strohaber, C. Petersen, and C. J. G. J. Uiterwaal, Opt. Lett. 32, 2387 (2007). 